\title{
Linx
}

Revue des linguistes de l'université Paris X Nanterre

$12 \mid 2002$

« Comme la lettre dit la vie »

\section{Autour de la référence : désignatifs et termes d'adresse en moyen français}

Dominique Lagorgette

\section{(2) OpenEdition}

\section{Journals}

Édition électronique

URL : http://journals.openedition.org/linx/1289

DOI : 10.4000/linx.1289

ISSN : 2118-9692

Éditeur

Presses universitaires de Paris Nanterre

\section{Édition imprimée}

Date de publication : 1 octobre 2002

Pagination : 106-117

ISSN : 0246-8743

\section{Référence électronique}

Dominique Lagorgette, «Autour de la référence : désignatifs et termes d'adresse en moyen français », Linx [En ligne], 12 | 2002, mis en ligne le 10 octobre 2012, consulté le 01 mai 2019. URL : http:// journals.openedition.org/linx/1289; DOI : 10.4000/linx.1289

Ce document a été généré automatiquement le 1 mai 2019.

Département de Sciences du langage, Université Paris Ouest 


\title{
Autour de la référence : désignatifs et termes d'adresse en moyen français
}

\author{
Dominique Lagorgette
}
«Tiens, c'est marrant, ces trucs-là ! On dirait qu'il y en a toutes les fois qu'on va demander quelque chose à quelqu'un. Comment ça s'appelle? - Des appellatifs.- Comme ceux en apostrophe? Mais c'est pas pareil! - Ça ferait un bon sujet pour un D.E.A.... » Entendu, Séminaire de D.E.A. de Michèle Perret, Paris X - Nanterre, avril 1989.

Merci, Michèle, d'avoir cru en cette curiosité.

1 A partir de récits brefs des $14^{\mathrm{e}}$ et $15^{\mathrm{e}}$ siècles, les XV Joyes de Mariage et les Cent Nouvelles Nouvelles, nous tenterons de dégager les grandes lignes régissant la construction de la référence par le GN, en appuyant notre réflexion sur la grammaire textuelle et les théories de l'énonciation, qui ont pris source dans les travaux d'Emile Benveniste (1966; 1974). Notre propos est de montrer comment une étude linguistique d'un corpus diachronique littéraire peut permettre de mieux comprendre les effets sémantiques ou pragmatiques créés par le narrateur, à travers l'étude des énoncés des locuteurs. Nous opposerons les GN désignatifs (sujet ou objet) dans le récit aux termes d'adresse, syntagmes nominaux détachés dans le discours ${ }^{1}$. Nous proposons de définir les désignatifs comme les syntagmes nominaux de dénomination et de désignation des personnages. Les termes d'adresse sont des groupes nominaux renvoyant exclusivement à la deuxième personne (l'allocutaire) dans le discours direct.

2 Nous verrons tout d'abord comment les GNDés apparaissent dans les nouvelles², puis quels effets ils produisent, pour finir par une confrontation des GNDés et des T.A. 


\section{Désignatifs et termes d'adresse : construction de la référence}

3 A la suite de Francis Corblin (1995), nous dirons que les syntagmes nominaux sont les éléments privilégiés de la construction de la référence. Dans ce corpus, le passage du récit au discours direct est particulièrement significatif. Les narrateurs des XVJ et des CNN tendent à utiliser l'insertion du discours rapporté comme des témoignages dans un procès.

\subsection{Les noms propres}

4 Les XVJ et les CNN présentent des stéréotypes,des personnages bien connus du lectorat puisqu'il s'agit de bourgeois, de nobles, de membres du clergé ou de paysans dont les profils sont déjà présents dans les fabliaux du $13^{\mathrm{e}}$ siècle. Parmi ces $\mathrm{GN}$, quelques cas sont rendus uniques par des NP : ce fait est traité comme rare par la plupart des chercheurs ${ }^{3}$. Pourtant, ce phénomène apparaît dans 18 récits sur cent. On attribue d'ordinaire ceci à une volonté de marquer surtout le statut social du personnage. Nous nous proposons d'invalider ce point de vue, en particulier avec les CNN. La manière d'introduire les NP dans le récit n'est, en effet, pas homogène, comme nous allons le voir.

Ainsi, le narrateur de la Nouvelle 24, Monseigneur de Fiennes, en commençant son récit, procède-t-il par nomination abrupte, rompant la règle d'or du recueil, qui consiste à dissimuler les noms propres des personnages - ce dont il est tout à fait conscient :

(1) «Jasoit que es nouvelles desusdictes les noms de ceulx et celles a qui elles ont touché et touchent ne soient mis n'escripz, si me donne mon appétit grand vouloir de nommer, en ma petite ratelée, le conte Walerant, en son temps conte de Saint Pol, et appellé le beau conte. » ( CNN 24, 1.4-9).

Ce NP, vu son emploi, suggère que le comte Walerant est un personnage connu du cercle des auditeurs-conteurs-lecteurs mis en scène par l'histoire-cadre ${ }^{4}$. On précise qu'il est seigneur d'un village « près dudit Lisle » (l.11), ce qui l'inclut dans le Duché de Bourgogne, lieu même des narrations. Il appartient donc aux personnages célèbres, dont la connaissance est commune au cercle, comme à toute la France puisqu'il est en fait un des héros de la Guerre de Cent Ans, apparaissant régulièrement dans les Chroniques de Froissart ${ }^{5}$.

7 De même, Monseigneur Talebot, dans la Nouvelle 5, fait l'objet de ce type de référence ex nihilo:

(2) «Monseigneur Talebot, a qui Dieu pardoint, capitaine anglois si preux, si vaillant, et aux armes si eureux, comme chacun scet, fist en sa vie deux jugemens dignes d'estre recitez et en audience et memoire perpetuelle amenez. » (CNN, 5, 1.4-8).

8 Le public se doit de connaître le personnage (comme chacun scet), et si ce n'est pas le cas, la nouvelle se chargera de l'informer (on notera le paradoxe). Il est lui aussi mentionné par Froissart.

9 Comme le précise Michèle Perret (1995 : 178), on parlera de nomination abrupte lorsque l'on se trouve face, comme dans ces deux cas, à :

[une] référence situationnelle à l'état pur, qui fait appel à la connivence absolue de

l'interlocuteur. Cette nomination abrupte repose en effet sur la présomption que le 
nom propre jeté dans la conversation sera immédiatement identifié par l'interlocuteur parce que célèbre pour lui.

10 Si l'on compare cet emploi du NP dans les Nouvelles 5 et 24 avec celui des autres CNN où des NP sont mentionnés, on mesure alors toute la distance qui existe entre ces différents types de référence :

(3) «Si fist partir la compaignie; et au partir que firent les femmes dirent dame Jehanne, dame Ysabeau et Katherine: "Helas! musnier, que vous serez bon home si vous faictes revenir ce dyamant. " (CNN, 3, 1.230-233).

(4) « A l'ostel d'un grand baron du païs demouroit et residoit ung jeune, gent et gracieux gentilhomme, nommé Gerard, qui s'enamoura tresfort d'une damoiselle de leans [en la duché de Brabant] nommee Katherine. " (CNN, 26, 1.10-13).

(5) « Et entre les autres nobles hommes avoit ung qui se nommoit Jehan Stotton, escuier trenchant, et Thomas Brampton, eschanson dudit cardinal, lesquels Jehan et Thomas Brampton se entreaymoient autant ou plus que pourroient faire deux freres germains ensemble. »(CNN, 62, 1.15-20).

(6) « Et comme ilz estoient ou meilleur endroit de leur disner et qu'ilz faisoient le plus grand het, veez cy leens venir ung homme qui s'appelle Trenchecoille, lequel se mesle de tailler gens, d'arracher dens, et d'un grand tas d'aultres brouilleries. » (CNN, 64, 1.18-23).

11 Les manières d'introduire un ou plusieurs personnages varient selon l'importance qu'il (ou elle) aura dans l'histoire : ainsi dans la Nouvelle 3, les trois femmes sont simplement mentionnées par la compaignie puis les femmes, tandis que Gérard et Katherine (CNN 26), Jehan Stotton et Thomas Brampton (CNN 62) puis Trenchecouille ( $C N N$ 64) font l'objet de présentations plus complètes.

12 Dans les deux derniers cas, la présentation indéfinie est minimale, et les précisions suivent le NP. Dans le cas de Trenchecouille, le narrateur a recours à une référence visuelle (1.20: "veez cy leens venir»), qui opère un embrayage contextuel, marquant fortement sa situation de témoin comme étant le point de départ spatio-temporel du récit : sa connaissance du personnage va devenir commune aux différents « témoins » de l'histoire. La caractérisation qui suit en est le moyen.

On parlera pour ces trois derniers exemples (CNN 26, 62 et 64) de nomination après présentation indéfinie, qui, d'après M.Perret (1995: 180) :

consiste à introduire un personnage par l'indéfini et à lui attribuer ensuite un nom propre, soit directement, soit à l'aide d'un verbe de nomination (estre apelé, avoir a nom).»

14 Un autre cas de dénomination se présente dans la Nouvelle 63, avec Montbléru, qui est lui-même narrateur et commence ainsi :

(7)« LA SOIXANTE TROISIEME NOUVELLE par MONTBLERU Montbleru se trouva, environ deux ans a, a la foyre d'Envers, en la compaignie de monseigneur d'Estampes, qui le deffrayoit, qui est une chose qu'il prend assez bien en gré. » (CNN, 63, 1.1-7).

On remarque ici un cas particulièrement intéressant où le narrateur de l'histoire coïncide avec le personnage principal sans toutefois que le récit ne soit formulé à la première personne. On pourrait parler, dans cet exemple, d'une illustration très particulière de référence égocentrique, dans la mesure où le narrateur ne se présente pas, mais construit la référence à partir de lui-même. La référence égocentrique est présentée par M.Perret (1995) comme « un ancrage référentiel par rapport au je du narrateur ».

Dernier type de référence par le NP, enfin, lorsque le NP des personnages est mentionné pour la première fois lors de passages de discours direct: alors que tout le réseau 
anaphorique dans le récit jusqu'à ce point a renvoyé à des descriptions indéfinies puis définies.

Il peut s'agir de GNdés employés pour référer à un délocuté :

(8)« Et a qui le payez vous ? dit il. - A frere Eustace. (...) Frere Bartholomeu en a autant ou plus.. (...) Frere Jacques aussi en a beaucop, et frere Anthoine aussi.» (CNN, 32, 1.106-130).

Mais dans la plupart des cas, il s'agit de T.A. dans le discours direct, qui sont ensuite repris comme GNdés dans le discours direct d'un autre personnage :

(9)« "Dy je pas bien, Jehannette ?” " dit il a la damoiselle dessus dicte. » (...) - « A dya, dit madame, monseigneur...(...) Si vous lairray Jehannette qui vous entretiendra.. » (CNN, 39, 1.109-116).

ou dans les sections contenant des verbes de parole (en fin de narration ou en incise) :

(10 «Et en ce faisant commence a dire la nonnain: "Pardieu, mon amy frere Aubry, je veil bien...(...) - Par ma foy, dist frere Aubry, seur Jehanne m'amye, je cognois ce que vous dictes." ")(CNN, 46, 1.91-98).

(11)« Elle commence a hucher tant qu'elle peut : "Hau! Hacquin !Hacquin!" Et il respond : "Que veulx tu? que veulx tu? - Vien ça, vien ça, dit elle, si feras [cela]." Et Hacquin, qui en avoit son saoul luy respondit (..)» (CNN, 82, 1.37-41).

M. Perret (1995 : 185) mentionne ce type de référence dans le cadre de son analyse de Mélusine, et les nomme références en discours rapporté.

L'intertextualité et l'appartenance au même groupe culturel sont aussi des critères fondamentaux qui ne doivent pas être négligés lorsque l'on s'intéresse aux statuts des GNdés et des termes d'adresse dans les textes. Ce phénomène dépasse largement le domaine de la fiction, puisqu'il existe de nombreux traités destinés à faire comprendre les implications des choix de prénoms sur la personnalité de leur possesseur ${ }^{6}$. Le processus est facilement reconnaissable : là où le monde réel métaphorise la notion de destin, de fatalité, le récit fictionnel réemploie les mêmes présupposés directement, par l'usage des NP. Ce traitement des NP serait à rapprocher des stéréotypes, qui ont un rendement bien plus élevé qu'on ne le pense dans le domaine fictionnel.

21 Dans le même ordre d'idées, l'emploi du NP dans les XVJ renvoie à un stéréotype, extérieur au texte ou au contraire créé dans ses limites. Ainsi, Jehanne/Jouhanne est systématiquement employé pour une servante ${ }^{7}$. Il s'agit plus de référer à une fonction qu'à une personne, aussi ce personnage ne fait-il pas l'objet d'une description psychologique particulière. Là n'est pas le but du texte, qui ne cherche nullement à construire des personnages. Comme tout traité de morale, les XVJ réduisent leurs personnages à leurs actes, et chaque énoncé prend valeur d'action édifiante, illustrant les dires du narrateur. Car l'enjeu même du recueil est là : fournir des planches descriptives d'un itinéraire moral, sensiblement proches des planches d'anatomie - une anatomie du mariage, en quelque sorte.

Le stéréotype donne un cadre minimaliste et économique, qui permet de n'avoir pas à bâtir de description psychologique, ce qui est extrêmement important dans un genre qui se donne pour contrainte principale la brièveté. La reconnaissance du stéréotype par le lecteur est un point crucial pour que fonctionne la structure narrative, et la répétition des mêmes éléments de description dans les recueils vient renforcer ce procédé de décodage. 


\subsection{Les noms communs}

\subsubsection{Les termes occupationnels}

Les termes occupationnels foisonnent en GNDés dans les récits brefs, en particulier dans les CNN, ainsi qu'en témoigne ce relevé (non exhaustif) des métiers mentionnés pour les personnages principaux : une chamberiere (XVJ, $C N N)$, une bergiere (CNN), une muniere (CNN 3), l'enfermiere (CNN 21), la bouchiere (CNN40), un escuier (CNN 18;31;72), un serviteur (CNN 27), un compaignon (CNN $1 ; 34 ; 37 ; 59 ; 72 ; 75 ; 97)$, un marchant (CNN $38 ; 43 ; 49 ; 73 ; 99)$, le curé (CNN $44 ; 64 ; 73 ; 85 ; 94 ; 96)$, un clerc (CNN $13 ; 23 ; 41 ; 42 ; 93 ; 99)$, bon evesque (CNN 100), ung grand clerc et prescheur de l'ordre saint Dominicque (CNN 40), un chevalier (CNN $3 ; 9$; $31 ; 33 ; 35 ; 36 ; 39 ; 54 ; 70 ; 76 ; 98)$, notre president (CNN 47).

Or ces termes apparaissent rarement en adresse. Plutôt que de penser avec A. Zwicky (1974) et J.-Cl. Milner (1978) que ce constat les exclut de la classe des termes d'adresse, nous dirons que leur fonction se limite souvent au récit parce qu'elle est de fixer pour le lecteur le rôle social du personnage, ce qui pourrait dès lors renvoyer à un type social avec toutes ses caractéristiques d'usage dans le groupe considéré. On pourrait interpeller quelqu'un par sa fonction (et d'ailleurs, cela se produit dans le corpus), mais la politesse fait que l'on préfère user d'autres formes plus déférentes, si l'on choisit un titre, ou plus adéquates à la relation entretenue. En revanche, dans le récit, il est plus rapide de mentionner la fonction afin de faire l'économie d'une description si cette fonction a une quelconque pertinence dans l'histoire.

\subsubsection{Les GNDés des personnages principaux.}

Les GNDés qui introduisent les personnages dans les XVJ sont généralement rédigés dans un style économique, disant l'essentiel pour la suite de la démonstration, et pas un mot de plus (Ferrier, 1954: 28). La plupart des noms communs renvoyant aux personnages principaux de l'intrigue sont précédés du possessif (sa femme, sa dame), lorsque c'est le mari qui sert de point de repère, ou formés de la proposition plus complexe [det+(adv)+adj+nc]:

XVJ, III, $1.6:$ « le pouvre mary »; XVJ, III, $1.2:$ « le jeunes homs »

$X V J$, VII, $1.5:$ : une tres bonne galloise»

On trouve fort peu de groupes désignatifs élaborés (c'est-à-dire dépassant cette forme [det $+a d v+a d j+n c])$. Au contraire, on aura des désignatifs réduits au minimum: sexe, condition sociale et élément distinctif pertinent pour la suite du récit, comme dans l'exemple de la Septiesme Joye, où le mari sera cocu et où, donc, le tempérament sexuel de son épouse est déterminant.

Si l'on examine les Joyes où l'un des deux partenaires est décrit par rapport à l'autre, on trouve plus de cas où la femme est désignée par rapport à l'homme (sa femme: Joyes 1, 3, 4, 8, 13, soit 55\%) que l'inverse (son mary : Joyes 6, 7, 15 ; au mary : Deuxième Joye, soit 45\%).

Dans la mesure où l'homme est le principal dédicataire du texte, il y tient le rôle central et sert de référent. Dès lors, la femme n'a plus d'intérêt que dans la relation qu'elle entretient avec lui.

Il arrive aussi que certains personnages soient désignés de manière autonome sans référent marqué par le possessif, tant hommes que femmes. Ainsi se substitue à l'adjectif possessif l'article défini dans la majorité des cas, voire plus rarement l'article indéfini : 
Quinte Joye, 1.11-12 : « la dame / le proudomme »

Quinte Joye,1.34-38 : « le bonhomme / la bonne damoiselle ou bourgeoise »

Neuvième Joye, 1.2-4 : « la femme / le jeune homme »

Onzième Joye, 1.1-20 : « ung gentil galant / une belle damoiselle »

Remarquons la symétrie de construction de ces formes qui conservent la même sorte d'article d'un personnage à l'autre. Nous pouvons d'ores et déjà remarquer l'organisation binaire des désignatifs, qui fonctionnent apparemment par paires symétriques ou par dyades , pour reprendre la terminologie dont usent Brown et Ford (1961: 234) afin de caractériser les termes d'adresse.

Une dyade est composée de deux branches: locuteur et allocutaire, avec des échanges de places dans le cours de l'interaction verbale. Chaque branche peut comporter de un à ${ }^{n}$ locuteurs, du moment qu'ils représentent la même idée. Ainsi, on parlera d'une seule dyade lorsque, dans la Nouvelle 80, le jeune époux se trouve confronté à la famille de sa femme, résumée dans le groupe désignatif correspondant par des (séries de) termes comme : « ung grant tas des amys d'elle " (1.41-42) ou " sa mere, sa seur, sa tante, sa cousine, sa voisine » (1.50-51). De même, on parlera d'une dyade lorsque l'on sera en présence d'un mari et de sa femme, par exemple.

En ce qui concerne les $C N N$, la femme y est fréquemment présentée comme une entité autonome, sans passer par son lien aux autres personnages. Dans ces cas-là, elle est en fait décrite selon trois types de critères : son statut social, son apparence et son caractère ${ }^{8}$.

\begin{tabular}{|l|l|l|}
\hline statut social & apparence & personnalité \\
\hline la femme $(85,91)$ & une belle fille $(22,25,55)$ & $\begin{array}{l}\text { une bonne et loyale femme } \\
(59)\end{array}$ \\
\hline les femmes (32) & une tres belle fille (24) & une vaillant femme (61) \\
\hline une femme mariee (68) & une jeune fille (28,53) & la plus devoiee femme (84) \\
\hline une gente femme mariee (93) & une tres belle jeune fille (43) & $\begin{array}{l}\text { une notable et vaillant } \\
\text { femme(34) }\end{array}$ \\
\hline $\begin{array}{l}\text { une damoiselle de Maubeuge } \\
(54)\end{array}$ & une tresbelle femme (66) & femme aultre que bonne (47) \\
\hline $\begin{array}{l}\text { une ancienne damoiselle } \\
\text { vefve (83) }\end{array}$ & $\begin{array}{l}\text { une femme belle et en grand } \\
\text { point (73) }\end{array}$ & \\
\hline \hline \begin{tabular}{l} 
la chambriere de leans (18) \\
\hline
\end{tabular} & & \\
\hline
\end{tabular}

\begin{tabular}{|l|l|l|}
\hline une jeune pastorelle (8) & une belle fille et bonne (20) \\
\hline & une belle, gente et jeune femme (3) & une belle damoiselle et bonne (36) \\
\hline & une plaisante et assez gente femme (88) & une tendre jeune fille (86) \\
\hline & une donzelle belle et gente (76) & \\
\hline
\end{tabular}




\begin{tabular}{|l|l|l|}
\hline & une belle damoiselle (33) & \\
\hline une tresbelle damoiselle $(27,31,35)$ & \\
\hline une tresbelle et gente damoiselle (41) & \\
\hline une tresbelle et gente damoiselle mariée et mercière (4) & \\
\hline une tresbelle jeune et gente fille a marier (44) & \\
\hline une tresbelle femmelette jeune et en bon point(95) & \\
\hline une tresbelle dame (39) & \\
\hline une tresbelle, gente et jeune dame (81) & \\
\hline & une tresbelle dame et de haut lieu (16) & \\
\hline & une tresbelle jeune chambriere (87) & \\
\hline
\end{tabular}

33 On remarque, d'après ce tableau, que la plupart des désignatifs renvoient à plusieurs catégories et en particulier au statut social et à l'apparence globale de la personne ${ }^{9}$.

34 Les personnages féminins mentionnés ne font pas l'objet de descriptions plus poussées pour préciser leur apparence physique. Elles restent toutes en suspens, en quelque sorte, car on sait simplement qu'elles sont belles ou bien en point, deux critères totalement subjectifs. De même pour les traits de caractère.

35 Seuls renseignements véritablement objectifs, les éléments renvoyant au statut social apportent des précisions sur le milieu dans lequel se déroule l'histoire; voilà peut-être pourquoi l'on trouve des titres comme dame ou damoiselle, s'opposant à bourgeoise, fille ou chambriere et pastorelle. Ces éléments en effet ne servent pas seulement à décrire le personnage mais favorisent l'implantation d'un décor autour de l'intrigue ${ }^{10}$. Damoiselle renvoie à un milieu socialement élevé, tandis que pastorelle évoque le monde rural des petites gens. Les désignatifs ont donc une fonction descriptive par rapport au personnage auquel ils s'appliquent ainsi que par rapport à la mise en place du contexte de l'histoire elle-même. Ils plantent le décor, en quelque sorte.

36 Les désignatifs portant sur la personnalité du personnage sont moins nombreux que les autres (8 sur 42, soit 19\%). Sur ces huit groupes nominaux, deux seulement sont connotés négativement (soit presque 5\%). Nous ne devons pas en déduire que les femmes sont toujours des anges dans ce recueil, mais tout simplement que ce sont les hommes, victimes, qui servent de point de référence d'ordinaire.

37 Les femmes mentionnées dans les autres nouvelles le sont par rapport à un homme qui luimême fait l'objet d'un désignatif, comme dans les XV Joyes. Leurs qualités ou défauts sont déductibles de la description de leur pivot référentiel et des relations qu'elles entretiennent avec lui. Ainsi, un homme décrit comme bon et dont l'épouse ne bénéficie que d'un sa femme pour toute description est souvent la dupe de cette moitié. 

personnage faisant l'objet d'un groupe nominal désignatif complexe a toujours un rôle crucial dans la nouvelle. Il est celui par rapport auquel tous les personnages sont décrits et son aventure prime. Il est en quelque sorte mis en relief par les GNDés qui jouent le rôle de projecteurs, de "poursuites", et pointent l'attention vers cet objet. Les GNDés définis signalent que le personnage dont il est question est crucial dans l'histoire. Passons maintenant à l'étude de GNDés particuliers.

\subsubsection{Les désignatifs de groupe et d'anonymes}

Les GNDés de groupe comme : ung grand tas de gentilz compaignons (CNN 66), la brigade (CNN 68), ces bonnes gens (CNN 89), ces deux compaignes (CNN 92), ces dyables de cordeliers (CNN30) sont rarement ensuite employés en adresse. La politesse peut être une explication envisageable, puisqu'on s'adresse plutôt à des allocutaires connus qu’à des indénombrables.

Les $C N N$ et les XVJ tendent à particulariser les personnages plutôt qu'à les amalgamer : ainsi, le groupe des femmes qui prennent la parole dans la Nouvelle 80, 1.50-51: « sa mere, sa seur, sa tante, sa cousine, sa voisine, » est divisé en sous-catégories, donnant une impression de foisonnement. Mais les paroles qui suivent ces GNdés sont organisées en phrases tout à fait cohérentes, comme si un seul individu avait pris la parole, et on voit bien l'intention du narrateur, qui tente de suggérer que tout un groupe de femmes se met à parler d'un seul coup.

\subsubsection{Les axiologiques négatifs}

On notera que les axiologiques négatifs dans les récits brefs en désignatifs sont relativement fréquents. Les Quinze Joyes de Mariage en usent avec parcimonie. En revanche, les Cent Nouvelles Nouvelles ne lésinent pas dans leur usage des insultes pour désigner les personnages négatifs parfois, mais surtout pour faire état de propos rapportés au style indirect.

On retrouve la plupart des T.A. en désignatifs, mais comme toujours, les syntagmes nominaux de désignation font l'objet d'expansions. Ainsi, dès la Première Nouvelle, les axiologiques négatifs apparaissent dans une séquence de discours indirect :

(12)«Dieu scet s'il parla hault et blasonna bien les armes de son bon voisin. Or l'appelle ribauld, après loudier, après putier, après yvroigne ; et tant bien le baptise que tous ceulx de la chambre et luy avec s'en rioient bien fort. Mais sa femme a ceste heure n'avoit pas ce loisir... » (CNN, 1, 1.94-95).

Ces groupes nominaux désignatifs sont particulièrement intéressants puisque ce sont d'anciens T.A., comme le verbe de parole appeler le souligne. Lorsqu'ils furent prononcés, ces mots étaient en adresse, et apparemment organisés en une série de quatre insultes ; la présence de après dans le discours indirect fait que l'on suit l'ordre chronologique d'énonciation des axiologiques, ce qui insiste sur l'oralité initiale de l'énoncé. Lorsque ces insultes nous sont rapportées, elles sont retransmises par le narrateur témoin, qui revendique ainsi sa présence, lors de l'énonciation, comme le verbe de parole au présent le marque clairement. Le narrateur crée de la sorte un curieux effet de décalage, puisqu'en employant le biais du discours indirect il souligne qu'il est le relais entre la scène et le lecteur, d'une part, tandis que d'autre part la variété des axiologiques 
(apparemment retranscrits fidèlement puisque dans un ordre particulier) et l'emploi du présent de narration confèrent à l'épisode une valeur de reportage en direct. Ce décalage est encore renforcé par l'environnement temporel de cette courte séquence de discours indirect : les autres verbes du récit sont en effet tous au passé (passé simple et imparfait se partagent la nouvelle).

La Nouvelle 4, au contraire, montre une intervention de l'auteur qui use d'un axiologique pour influer sur l'opinion du lecteur : «le pouvre coux de la ruelle» (1.90). Le mari caché par terre entre le mur et le lit de sa femme pour y prendre sur le fait un soupirant est caractérisé par un terme qui attire la compassion, dans un premier temps, mais qui finit, vu la suite des événements (sa totale lâcheté l'empêchant de sortir de sa cachette, il préfère sauvegarder sa vie plutôt que son honneur et n'intervient pas tandis que les affaires de l'amant avancent en besogne), par prendre une valeur tout à fait méprisante de la part du narrateur. Cette valeur négative de "pouvre " est confirmée 1.117 lorsque le mari devient «le pouvre martir», groupe nominal dont la connotation ironique est indéniable, dans une société courtoise où l'on peut imaginer que l'honneur soit une notion primordiale. Cette analyse vaut aussi pour la Nouvelle 75 , où il est question de « nostre pouvre coquart » (1.80).

Dernier cas de figure, enfin: lorsqu'un personnage dans le cours du discours direct mentionne un délocuté de manière négative. Ainsi, dans la Nouvelle 31, la demoiselle évoque un indélicat: "Le traistre et desloyal chevalier qu'il est se trompe il en ce point de moy?». De même, dans la Nouvelle 34, un mari soupçonneux, après avoir insulté sa femme, lui demande : "Ou est vostre houillier? », tout comme dans la Nouvelle 61 où le mari trompé rapporte l'histoire de l'infidélité de sa femme à ses beaux-parents et parle du « ribauld qui ce deshonneur nous a fait» (1.125). On retrouve dans cette même nouvelle deux autres occurrences de ce désignatif (l.125 et l.170).

Il semble toutefois que les GNdés axiologiques négatifs soient d'un faible rendement par rapport aux axiologiques négatifs en adresse. La plupart du temps, ils apparaissent avant ou après une section en discours direct comportant des T.A.[+axioneg]. L'insulte est plus fortement rattachée au discours qu'au récit.

\section{GN et embrayage}

Les termes d'adresse sont fréquemment ce que R. Jakobson (1963: 178) nomme des embrayeurs, dans la mesure où ils sont l'expression des relations entretenues entre le locuteur et l'allocutaire au moment où ils se parlent. Une classe de termes d'adresse est particulièrement fertile en ce domaine : ce sont les termes plus connus des anglophones sous le nom de kinship terms, ou « termes familiaux ». Dire « mon père » ou « mère » opère en effet un ancrage du discours dans la situation énonciative ainsi que dans son contexte.

De même avec «mon amy », «mon amie » et tout autre terme d'adresse recourant au possessif, notamment «ma dame», qui renvoient forcément au locuteur. Les termes d'adresse sont donc des actualisateurs, qui rattachent la partie dialoguée au locuteur, renvoyant ainsi à la première ou à la seconde personne. Le fait même que le terme d'adresse se limite à la deuxième personne du discours et exclue la non personne est un indice fort de l'embrayage qui peut être opéré par les termes d'adresse de type relationnels ou familiaux. Le locuteur qui dit je et interpelle autrui sous un terme qui lui est propre donne en même temps sa définition des rapports qu'il entretient avec l'allocutaire au moment de la prise de parole. 
50 Avec M.A.K. Halliday et R. Hasan ${ }^{11}$, on ajoutera à cette catégorie des embrayeurs les axiologiques négatifs, qui engagent l'opinion du locuteur sur l'allocutaire. En effet, si l'on insulte quelqu'un, c'est que l'on exprime un jugement personnel, assumé par je qui parle. Le statut même du T.A. est en fait l'enjeu de l'insulte: le locuteur souhaite que le T.A. axiologique négatif soit pris comme objectif, universel - en un mot, utilisable par tout autre locuteur pour interpeller l'allocutaire, comme un NP ou un titre pourraient le faire, ce qu'explicite bien (12) avec l'emploi de « baptiser »; en revanche, l'allocutaire, s'il interprète le T.A. comme une insulte, le percevra comme un embrayeur, renvoyant à la parole du locuteur, et à elle seule.

51 A la suite de Michèle Perret ${ }^{12}$, nous garderons la distinction entre embrayeur complet (saturé) et embrayeur incomplet (lacunaire). Nous classerons les termes d'adresse de type relationnel, familial et axiologique négatif dans la catégorie des embrayeurs saturés, puisque leur seule énonciation permet d'identifier le référent. En revanche, les termes d'adresse de type occupationnel, titres et nom propre posent un problème car ils visent une universalité plus ou moins objective. Pour interpeller un allocutaire: "Monseigneur le prévost », « Madamoiselle » ou « Montbléru », il suffit de connaitre le statut de l'allocutaire ; la position du locuteur n'est pas centrale dans l'énoncé, car ces trois catégories n'impliquent pas le locuteur à un niveau personnel.

52 Certains GNdés (que l'on ne trouve pas en adresse, comme la logique le commande) : le premier venu/le derenier venu, dans la Nouvelle 33, marquent une autre forme d'embrayage, interne à la logique du texte, où l'un/l'autre garantissent les relais anaphoriques. Leur seul intérêt dans la nouvelle est leur alternance dans la vie de la dame et non leur personnalité propre, malgré un effort du narrateur pour leur donner à chacun un style radicalement différent dans leurs discours.

53 Le premier venu l'est dans la chronologie de l'histoire racontée comme dans l'ordre d'apparition des personnages dans le récit. Il y a donc une superposition des deux niveaux où l'embrayage coïncide avec le cadre textuel (le contexte du locuteur (le narrateur)) et le contexte du délocuté (la femme ayant deux amants, classés chronologiquement par rapport au $\mathrm{T}_{0}$ de la première rencontre $: \mathrm{V}_{1}$ et $\mathrm{V}_{2}$ ). Cette coïncidence des plans marque un embrayage dans la mesure où la chronologie du récit s'aligne sur celle des événements historiques qu'elle rapporte. Toutefois, il est très clair que la dénomination est propre au narrateur (on imagine mal l'héroïne appeler ainsi ses deux amants..). On parlera ici d'un embrayage textuel, que l'on opposera à l'embrayage historique (celui du réel extérieur au texte). Le contexte du récit bref, soutenu par une histoire-cadre, rend seul possible ce type de situation : c'est uniquement parce que les histoires sont censées être vraies que l'on peut ainsi distinguer ces deux niveaux. Si l'auteur ne revendiquait pas sa fonction de simple relais, il n'y aurait qu'un embrayage textuel, ainsi que cela se produit dans les autres récits fictionnels. Les formes d'embrayage qui nous intéressent marquent une intervention de l'auteur. Ce type d'intervention est extrêmement fréquent dans les récits brefs. 


\section{Correspondances entre GNDés et termes d'adresse}

\subsection{Les $\mathrm{GN}$ de dénomination fonctionnent par dyades}

\subsubsection{GNDés}

Les GNDés tels qu'ils apparaissent dans le corpus se présentent souvent sous forme de paires symétriques ou dyades. On notera que les plus fréquentes sont celles qui mettent en présence les personnages principaux qui prennent ensuite la parole dans le discours direct (les renvois aux histoires ne sont pas exhaustifs):

XVJ, I, le mary / la dame ; XVJ, I, l'homme / la femme ; XVJ, V, l'amy / la dame

XVJ, III, la dame / la commere ; XVJ, III, le mari / le compere ;

CNN, 14, la mere / la fille ; XVJ, 9, le pere / le fils ;

CNN, 3, le musnier (12 occ.)/ le seigneur ( 2 occ.) ; XVJ, 15, la dame / la chambriere

CNN, 6, le prieur (16 occ.)/ l'ivroigne (12 occ.) ; CNN, 46 : les moynes / les nonnains

CNN, 5, l'Anglois (12 occ.) / le François (13 occ.)

CNN, 26, Katherine (13 occ.) Conrard (22 occ.)/ Gérard (36 occ.)

CNN,62, le dit Jehan Stotton (8 occ.)/ le dit Thomas Brampton (2 occ.)

CNN , 16, monseigneur (21 occurrences) / madame (19 occurrences)

On met donc en dyades des GNDés de même catégorie : nc[+occ] / nc[+occ] , NP/ NP, etc.. On retrouve pour les T.A. les mêmes dyades de personnages.

De plus, les mêmes adjectifs et plus généralement les mêmes structures sont fréquemment employés pour les membres des dyades. Ainsi, à titre indicatif :

CNN, 34, 1.39 : bon compaignon / 1.77 : bon mary ; CNN 31, 1.41 : ce bon chevalier / 1 .

46 : ce bon escuier

CNN, 59, 1.6: une bonne et loyale femme / 1.25 : le bon et loyal servant.

CNN, 16, 1.4 : un gentil chevalier / 1.31 : ung gentil escuier

CNN, 66, 1.9 : une tresbelle femme / $1.10:$ un tresbeau filz

Si les qualificatifs sont différents, il arrive alors que la structure soit similaire :

CNN, 27, 1.11: un tresgentil homme / 1.14 : une tresbelle damoiselle

CNN, 48, 1.4 : ung gentil compaignon / 1.4 : une jeune damoiselle

CNN, 53, 1.15 : ung homme ancien / 1.15 : une femme vieille

CNN, 33, le premier venu (12 occ.)/ le derrenier venu (12 occ.)

Enfin, des personnages sont souvent associés par des similitudes de construction qui soulignent en fait les différences et les oppositions entre les deux membres de la dyade :

$C N N, 53,1.31$ : le vieil homme riche et la jeune fille pouvre

$C N N, 53,1.33$ : le jeune homme pouvre / la vieille femme riche

On remarquera un jeu autour des GNDés dans cette même nouvelle qui montre combien l'ordre des mots est crucial lorsque la place de l'adjectif est en jeu, ainsi qu'en témoigne le chiasme :

CNN, 53, 1.42: «Si fut menée la pouvre jeune fille a l'ostel du riche homme.»

La vaste majorité des occurrences fait appel à la notion de pivot référentiel : un personnage est mentionné en fonction de sa relation à un autre; les rôles s'échangent et produisent un effet similaire à celui du "champ / contre-champ » au cinéma : le premier personnage est montré du point de vue du deuxième et le deuxième du point de vue du premier en alternance :

CNN, 4 : la damoiselle ( 1 occ.) / son mary (5 occ.) l'Escossois (9 occ.) / sa femme (4 occ.) 
CNN, 19 : ung bon et riche marchant de Londres / sa femme (7 occ.) la femme / son

mary (5 occ.)

CNN, 88 : le bon mary ( 2 occ.) / sa femme ( 2 occ.) ceste bonne bourgeoise / son

mary (6 occ.)

XVJ, V, la dame (20 occ.) / son mary (9 occ.) / son amy (9 occ.)

61 Les couples désignés par des adjectifs communs sont ainsi plus visibles pour le lecteur.

L'ajout du même adjectif met en relief complicité ou rapport de force. Cette symétrie est ellemême fréquente en adresse avec les dyades qui reprennent les discours des principaux personnages, mis en avant par les dyades de désignatifs, comme dans les exemples suivants :

XVJ, V, m'amie (9 occ.) / mon amy (13 occ.) ; XVJ, XV, madame/ Jouhanne,

CNN, 16 : monseigneur (6 occ.)/ dame ( 2 occ.) ; CNN, 88 : mon mary / m'amye

Un couple de locuteurs est donc fréquemment désigné par le même adjectif et cette situation fait ensuite l'objet de variations : l'absence de l'adjectif dans la progression du récit pour l'un des personnages, tandis que l'on voit son maintien pour l'autre, permet de suggérer une évolution des rapports de force et des rapports de places:

XVJ, IX, 1.2-4 : le jeune homme / la femme ;1.26-48 : le bon homme / la dame

1.49-78: le proudomme / sa femme ; 1.79-120 : le bon homme / la dame

$1.121-146$ : le proudhomme / la dame :

1.147-159 : le bon homme / Ø; 1.160-166 : le proudomme / Ø

Le narrateur peut grâce aux GNDés faire passer son opinion sur ses personnages à travers le $\mathrm{GN}$ au lieu de l'affirmer par l'emploi du GV, qui donnerait ces évolutions à voir de façon moins subtile. On voit bien dans ces exemples comment le personnage féminin ne change pas ou très peu de noms, tandis que les GNDés concernant le personnage masculin sont plus variables. On assiste même à l'effacement d'un des personnages, à la fin de la Neuviesme Joye.

Il arrive aussi souvent qu'un personnage soit décrit de manière très positive au début d'une nouvelle et que tout le récit s'applique en fait à transformer cette première approche en un véritable oxymore. On en trouvera un exemple dans l'évolution du personnage de Monseigneur Talebot, dans la Nouvelle 5, qui se conduit en fait de manière tout à fait désastreuse mais est au début présenté comme un être d'élite :

(13)« Monseigneur Talebot, a qui Dieu pardoint, capitaine anglois si preux, si vaillant, et aux armes si eureux... » $(\mathrm{CNN}, 5,1.4)$.

Quelques lignes plus loin, le titre disparaît, pour ne revenir que lorsque l'histoire a démontré la sottise de Talebot :

(14)«Et le pouvre Angloys s'en couroit par la chambre le plus qu'il povoit, et Talebot après, qui ...» $(\mathrm{CNN}, 5,1.114-115)$;

"A la verité, l'Anglois fut tant batu que presque jusques a la mort, et crya mercy a Talebot et au Françoys, qui par ce moien fut delivré, et de sa renson par monseigneur Talebot acquicté.» (1.118-120).

La conclusion de l'épisode met en relief ce décalage entre désignatifs et réalité, notamment en recourant à bon :

(15)« Veez la le premier jugement que fist le bon seigneur Talebot.» (CNN, 5, 1.124-125).

67 Pour reprendre ce que suggérait F. Corblin (1995), une analyse suivie des GNdés peut parfois suffire à résumer l'histoire, sans même examiner les SV. Ces dyades de personnages (les plus importants dans le récit au moment où la dyade se présente) peuvent comprendre tous les types de groupes nominaux que nous avons jusqu'alors décrits. Toutefois, la 
tendance, une fois de plus, est à l'économie et l'on trouvera surtout des dyades courtes (sans expansions du GN).

\subsubsection{T.A.}

parfois dans une même classe, comme dans ces exemples où les deux syntagmes renvoient à la catégorie relationnelle :

(16)« Lui demande : "Qu'avez vous, m'amye? - Vroiement, mon amy, j'ay si grant mal en ung cousté et ou ventre que c'est merveilles ; (...)-M'amie, fait il, tournez vous devers moy.Par Dieu, mon amy, fait el, je suis si chaude que c'est merveilles et ne peu ennuyt dormir. » ( XVJ, V, 1. 131-137).

Il arrive fréquemment que cette dyade s'opère d'une classe à l'autre, lorsque la hiérarchie est impliquée. Par exemple entre un noble et une paysanne, où le gentilhomme emploie les termes relationnels tandis que la meunière use du titre de politesse :

(17) « et luy dist: "Certes, ma'amye, j'apperçoy bien que vous estes malade et en grand peril ». Et a ces parolles la musniere s'approucha et dist: " Helas! monseigneur, et que me fault il ?- Vrayement, m'amye, j'apperçoy bien que si vous cheminez gueres avant, que vostre devant est en tresgrand dangier de cheoir ; (...) »)(CNN, 3, 1.22-29).

"Si respondit: "Helas! monseigneur, et que dictes vous? et a quoy cognoissez vous que mon devant est en dangier de cheoir? Il me semble qu'il tient tant bien. -Dya, m'amye, respondit monseigneur, suffise vous a tant, et soiez seure que je vous dy la verité... » (CNN, $3,1.36-42)$.

La plupart de ces catégories fusionnent au gré de l'imagination du locuteur, et l'on trouve ainsi ma tres chere compaigne et tres loyale espouse (CNN, 1, 1.209-210), ma bonne mere (CNN, 14, 1.108) etc.

\subsubsection{Dyades GNDés > dyades T.A ?}

71 Là où les GNDés fonctionnent par dyades, nous retrouvons donc les mêmes personnages couplés par dyades dans le discours direct. En fait, les dyades de GNDés préparent le passage au discours direct des principaux personnages, en constituant une structure de base à laquelle le lecteur a tout le temps de s'habituer pendant le récit. On crée de la sorte une attente qui est comblée par la prise de parole. Cette prise de parole apparaît alors comme une démonstration de tout ce que le récit insinuait ou affirmait quant aux rapports entre les différents personnages. Le fait de les voir parler ${ }^{13}$ renforce en effet les dires du narrateur. Cette analyse des structures dyadiques ne fonctionne donc pas seulement pour notre corpus de textes en moyen français, mais pour tout texte narratif en général, puisque l'on remarquerait exactement les mêmes structures dans des textes classiques, modernes ou contemporains. Le rapport mimétique à la parole est en fait la seule explication de cette constance structurelle.

Le recours aux dyades dans les GNDés et les termes d'adresse, avec la mise en relief graduelle du personnage en tant que tel, c'est-à-dire comme une entité à construire avec une psychologie qui se développe (versus l'histoire), est un pas vers le roman moderne, et nous sommes ici en désaccord avec l'ensemble des critiques qui considèrent que les personnages dans les nouvelles du corpus ne «se développent pas». Il arrive fréquemment au contraire qu'une histoire ait pour but de montrer comment une aventure advenue à un couple de personnages les a amenés à changer - ne serait-ce que 
leur nom, ainsi qu'en témoignent par exemple les Nouvelles 64 et 73, avec respectivement Trenchecouille et Sire Baudin Casier.

Le lecteur perd ses repères lorsqu'il s'agit de déterminer si le surnom est venu à Trenchecouille lors de l'histoire que l'on rapporte ou s'il en était déjà titulaire avant l'anecdote. Le texte est en effet peu clair sur ce point :

(18)« Et comme ilz estoient ou meilleur endroit de leur disner et qu'ilz faisoient le plus grand het, veez cy leens venir ung homme qui s'appelle Trenchecoille, lequel se mesle de tailler gens, d'arracher dens, et d'un grand tas d'aultres brouilleries » (CNN, 64, 1. 18-23).

74 A la lecture de cette présentation, il paraît clair que ce personnage porte réellement ce nom. Mais un peu plus loin, on retrouve cette appellation sous la forme d'un nom commun qualifié par un adjectif démonstratif puis l'article défini, et privé à chaque fois de majuscule ${ }^{14}$ :

(19)« Ce maistre curé (...) commence a prendre la parole a ce trenchecoille et luy va demander de son mestier et de mille choses; et le trenchecoille luy respondoit au propos le mieulx qu'il savoit » (CNN, 64, 1.28-32).

75 Toute la suite de la nouvelle présente ce type de reprises anaphoriques où trenchecoille apparaît comme un nom commun déterminé. On ne sait plus de fait s'il s'agit de son patronyme, d'un surnom ou d'une sorte de titre occupationnel. Cette confusion est renforcée par le décalage entre la première forme de présentation, introduite par le verbe « s'appeler ", dont l'ambiguïté reste forte à cette époque ${ }^{15}$, et les termes de reprise qui transforment ce nom propre en nom commun, de type occupationnel. Enfin, cette désignation est particulièrement ambiguë dans la mesure où, s'il s'agit d'anthroponymie, il faut remarquer que l'homme est déjà nommé avant que ne lui arrive l'aventure dont il tirera son nom. Mais même cet élément reste flou.

Enfin Sire Baudin Casier, qui sont les derniers mots de la Nouvelle 73, présente un cas similaire de dénomination née d'une mésaventure :

(20) «Et ne sçay point que son curé s'i trouvast depuis, lequel, au moien de ceste adventure,

fut, comme encores est, appellé Sire Baudin Casier. » (1.255-257). Le but de la Nouvelle 73 pourrait tout à fait être de narrer la genèse de cette dénomination.

Le fait que le discours direct renforce ou au contraire contredise la qualification des personnages dans le récit permet à l'auteur de donner au narrateur le rôle de narrateur omniscient ou au contraire de témoin d'une histoire qui lui échappe, dont il n'a pu prévoir la fin, comme le décalage apparent entre le récit et le discours dans leurs progressions vers la fin de l'intrigue tend à le montrer.

79 Les différents recueils de récits brefs adoptent à ce propos des stratégies variables, en accord avec leur but ultime.

\subsubsection{Effets de renforcement}

Dans la plupart des cas, dans les Quinze Joyes de Mariage, les dialogues servent de preuve au narrateur. Il a dépeint une femme cynique, menteuse et vile - le discours direct montre ce personnage dans ce type de situation face à son mari berné :

(21)« Le page du bon home est avecques les pages de ses amis en l'estable et leur compte comment la dame fait le malade (...) » (XVJ, VI, 1. $164-166)$.

"Quant vient au matin, [le mari] vient a sa femme et lui dit: "Vroiement, dame, je me merveille moult de vostre maniere ne je ne savroie comment me gouverner avecques vous. Ave Maria! fait elle. Et y a il tant affaire avecques moy? Hellas! fait elle, je ne fine jour et nuit de nourrir porcs, poulcins, oaies, et fille et travaille et fais le mieulx que je puis tant que je mourroy avant mes jours, et encore ne puis ge avoir une heure de patience! Et vous ne 
travaillez sinon a despendre et a gaster tout et a gens dont je n'ay que faire. - Que faire? fait

il. Ce sont gens qui me pouent bien nuire ou aider. » » (1.179-191).

Le dialogue sert en fait à emblématiser une situation type en s'appuyant sur un exemple, comme s'il s'agissait d'un guide des symptômes de malfaisance féminine. Le lecteur sait d'avance que le mari est trompé, bien souvent dans tous les sens du terme, puisque ce dernier est par définition le seul à ne jamais se douter de rien. Lecteur et narrateur se trouvent à égalité avec la dame, qui organise, ou participe tout au moins, aux mauvaises actions, tandis que le mari, pris comme exemplum en négatif, reste seul à tout ignorer. Dans la mesure où le texte se présente comme un traité moralisateur et didactique, on peut tout à fait imaginer que le lecteur puisse en fait se trouver face à lui-même, lorsqu'il reconnaît un contexte ou même des propos. A ce moment-là, les GNDés appliqués par l'auteur au personnage masculin prennent valeur de termes d'adresse pour le lecteur, s'il cherche réellement à identifier sa situation à celle du personnage. Voici qui pourrait expliquer partiellement pourquoi la désignation des personnages masculins est aussi compatissante, outre le simple fait que ce livre se donne comme narrataire les hommes en général. Le "poisson en la nasse " semble être un deuxième nom pour toute la gente masculine. C'est après tout le but affirmé du texte, dès son Introduction.

Les CNN, quant à elles, s'adressent à un autre public et ont un tout autre but. Lorsque ce procédé d'illustration se présente, il sert en fait simplement à animer une narration par quelques propos vifs, qui n'auraient pas été de mise dans le récit, ou n'auraient pas eu la même portée s'ils avaient émané du narrateur. On renforce de la sorte, preuve à l'appui, les descriptions du récit.

Plus souvent, pourtant, les GNDés et les termes d'adresse montrent un décalage qui produit des effets de sens.

\subsubsection{Effets de distanciation ironique} son mari en l'appelant «mon amy» tandis que l'amant est caché (sous les yeux du lecteur, en somme) à quelques mètres du cocu crée des effets de distanciation ironique ${ }^{16}$. La Septiesme Joye montre d'ailleurs jusqu'où peut aller cette distanciation puisque le mari lui-même montre sa crédulité, rapportée complaisamment par le narrateur :

(22)« (...) et auxi il n'est point a croire que la famme qui tant lui fait bonne chiere et le baise et accolle si doulcement et l'appelle "mon amy" peust jamés faire telle chouse [le tromper] et auxi il voit bien qu'il n'en est riens. » (XVJ, VII, 1.167-170).

5 La dame dans la suite de l'épisode multiplie les mon amy (10 occurrences entre les lignes 184 et 248, auxquelles on ajoutera : mon tres doulx seigneur et amy, 1.212, mon amy que je ame sur toutes chouses qui sont en terre, 1.215, et mon tres doulx amy, 1.238), alors que le lecteur sait, depuis les lignes 102 à 112, qu'en effet elle trompe son époux.

On remarquera, pour finir, que ce type de phénomène, loin d'être marginal, est constamment mis en œuvre dans les $C N N$, dans la mesure où il fait partie intégrante des ressorts comiques du recueil. Une même analyse pourrait d'ailleurs être appliquée à la plupart des œuvres de type récits brefs à caractère comique.

Il ressort de ces différents points que l'étude des GNDés amène rapidement à poser la question de la construction de la référence en moyen français en termes tout autant linguistiques que littéraires : comme nous l'avons à plusieurs reprises souligné, le type du texte amènera à évaluer les faits linguistiques de manières différentes. Un même groupe 
nominal prendra, selon son contexte d'occurrence (restreint mais aussi large), des valeurs sémantiques variables. D'où l'intérêt d'une étude des chaînes de référence en termes de grammaire textuelle matinée d'histoire littéraire : il nous semble en effet très difficile $\mathrm{d}$ 'isoler de tels faits de langue de leur contexte de production, puisque ce contexte inclut des contraintes liées à un certain type d'écriture. Langue et littérature sont de fait indissociables, puisque la seconde ne serait pas sans la première. Pour connaître la période médiévale, la linguistique a avant tout recours à des données littéraires. Qu'apprenons-nous, par les textes, de l'usage, des états de langue diachroniques? Même les textes didactiques, comme les Manieres de langage par exemple, ne peuvent se détacher du modèle littéraire, ainsi que nous en avons ailleurs formulé l'hypothèse ${ }^{17}$.

\section{BIBLIOGRAPHIE}

\section{Corpus}

Les Quinze Joyes de Mariage, éd. J.RYCHNER, Genève, Droz, 1967.

Les Cent Nouvelles Nouvelles, éd. F.P.SWEETSER, Genève, Droz, 1966.

\section{Texte consulté}

Manières de langage de 1396, 1399 et 1415, éd. A. M. KRISTOL, London, Anglo-norman Text Society (from Birkbeck College), 1995.

\section{Bibliographie}

CORBLIN, F. (1995) Les Formes de reprise dans le discours. Anaphores et chaînes de référence, Rennes : Presses Universitaires de Rennes.

DUFOURNET, J. (1995) « Faillite des valeurs et fuite du sens dans les Cent Nouvelles Nouvelles », Nord', 25 ; 41-50.

FERRIER, J. M. (1954) Forerunners of the french novel, an essay on the development of the nouvelle in the late middle ages, Manchester: Manchester University Press.

FERRIER, J. M. (1966) French prose writers of the 14th and 15th centuries, London: Pergamon Press.

HALLIDAY, M.A.K., HASAN, R. (1976) Cohesion in English, Londres : Longman.

JAKOBSON, R. (1953) Essais de linguistique générale, Paris : Ed. de Minuit, vol.1.

JEAY, M. (1992) Donner la parole. L'histoire-cadre dans les recueils de nouvelles des XV-XVI siècles, Le Moyen Français, 31, Montréal : Editions CERES.

LAGORGETTE, D. (1995) «Avoir (a) nom : étude diachronique de quelques expressions qui prédiquent le nom », LINX, $32 ; 113-132$.

LAGORGETTE, D. (1998) Désignatifs et termes d'adresse dans quelques textes en moyen français, Thèse dactylographiée, Paris 10 - Nanterre.

LAGORGETTE, D. (2001) « Prologues et épilogues dans quelques recueils de récits brefs en moyen français ", Bien dire, Bien aprandre, 19, 139-147. 
LAGORGETTE D. (en cours) «Termes d'adresse et verbes de parole en moyen français ».

LEWICKA, H. (1974, Etudes sur l'ancienne farce française, Paris : Klincksieck.

MILNER, J.-C. (1978) De la syntaxe à l'interprétation : quantités, insultes, exclamations, Paris : Le Seuil.

PERRET, M. (1988) Le Signe et la mention, Genève : Droz.

PERRET, M. (1995) « Histoire, nomination, référence », LINX 32, 173-188.

RASMUSSEN, J. (1958) La Prose narrative française du XV siècle, étude esthétique et stylistique,

Copenhague : Munksgaard.

ZWICKY, A. (1974) « Hey, whatsyourname ! », Chicago Linguistic Society, 10 ; 787-801.

\section{NOTES}

1. Pour une définition précise de ces deux notions, on se reportera à D. Lagorgette (1998: ch.1). Nous abrégerons dorénavant désignatifs par GNDés et termes d'adresse par T.A.

2. Dans la mesure où notre analyse porte sur les relations entre désignatifs et termes d'adresse, nous nous limiterons à l'étude des groupes désignatifs renvoyant à des personnages devenant sujets d'énonciation ou allocutaires dans le passage au discours direct. Nous avons donc exclu de notre corpus les Nouvelles 2, 11, 15, 45, 56, 58, 68, 69, 71, 74, 79, dans les Cent Nouvelles Nouvelles, ainsi que la $\mathrm{X}^{\mathrm{e}}$ et la XIV Joyes, où le discours direct, s'il était présent, ne comportait pas de T.A..

3. Voir par exemple J. Rasmussen (1958) ; J. Ferrier (1954), (1966).

4. Voir à ce sujet M. Jeay (1992), D. Lagorgette (2001).

5. Notons que le NP n'apparaît qu'en désignatif et jamais en adresse.

6. On peut prendre pour exemple de ce phénomène Un Prénom pour la vie, qui est connu des libraires comme un «best seller» toutes saisons confondues, notamment parmi le lectorat féminin en passe de donner le jour.

7. Voir Lewicka (1974 : 78-84).

8. Les numéros entre parenthèses renvoient aux Nouvelles.

9. Ceci va dans le sens des analyses de S. Lehmann, dans ce même volume.

10. De plus, on notera au passage que ces deux termes renvoient à deux archétypes littéraires différents: le roman courtois et la pastourelle, qui transfèrent ainsi instantanément les propriétés de leurs personnages sur ceux des CNN par cette allusion. Les Nouvelles pourraient en effet être envisagées comme des pastiches ou tout au moins des micro-réécritures des types plus nobles - phénomène de mise à distance ironique commune, quelle que soit l'époque, à la littérature dite "vulgaire», "populaire» ou "de gare», selon le degré de subjectivité du critique, ainsi qu'une étude, en cours à ce jour, le mettra en évidence.

11. M.A.K. Halliday et R. Hasan (1976:277).

12. M. Perret, (1988:31) : «Nous proposons de diviser les embrayeurs en deux types: - d'une part, les embrayeurs qu'on a appelés directs (M.Vuillaume) ou complets, transparents (G. Kleiber) : aujourd'hui, hier, je par exemple. A chacune de leurs occurrences, ces embrayeurs n'ont qu'un seul référent possible, si bien qu'il suffit de connaître les conditions de leur énonciation pour identifier leur référent ; - d'autre part, des embrayeurs indirects (M.Vuillaume) ou incomplets ou opaques (G. Kleiber), comme les démonstratifs, par exemple: «leur seule énonciation ne garantit pas l'identification du référent » (G. Kleiber, 1984, p.19). »

13. Cette image visuelle est à prendre au sens littéral, puisque l'on utilise le discours direct comme un document à l'appui dans un reportage : il faut dire le vrai.

14. Ce choix de l'éditeur est motivé vraisemblablement par la présence des déterminants. 
15. Pour un historique des expressions qui prédiquent le nom en ancien et moyen français, on se reportera à D. Lagorgette (1995).

16. Comme le remarque Jean Dufournet (1995: 43) au sujet des Cent Nouvelles Nouvelles : «La parodie concerne d'abord la présentation laudative des personnages, faite le plus souvent de l'accumulation de plusieurs adjectifs (...). Le jeu va plus loin quand la nouvelle apporte un démenti aux superlatifs initiaux ; la déception devient peu à peu une loi textuelle qui engendre le rire et requiert du lecteur une prise de recul critique par laquelle il fait mémoire de ce qui a été écrit. Elle peut intervenir très vite (...). Ailleurs, le déroulement de la nouvelle accomplit la dégradation d'un personnage initialement porté au pinacle." Ces lignes résument toutes les stratégies mises en œuvre par les narrateurs dans l'ensemble de leur écriture, mais fonctionnent tout autant pour le passage des GNdés aux termes d'adresse et vice versa.

17. Voir D. Lagorgette (en cours).

\section{AUTEUR}

\section{DOMINIQUE LAGORGETTE}

Université de Savoie 